\title{
Hypogravity research and educational parabolic flight activities conducted in Barcelona: a new hub of innovation in Europe.
}

\author{
Antoni Perez-Poch \\ ICE Education Sciences Institute; Dept. Computer Science; UPC, Universitat Politècnica de Catalunya, Spain, \\ antoni.perez-poch@upc.edu \\ Daniel Ventura González \\ Aeroclub Barcelona-Sabadell, Barcelona, Spain, daniel@estudio-brainstorm.es \\ David López \\ ICE Education Sciences Institute; Dept. Computers Architecture; UPC, Universitat Politècnica de Catalunya, \\ Spain, david@ac.upc.edu
}

\begin{abstract}
We report on different research and educational activities related to parabolic flights conducted in Barcelona since 2008. We use a CAP10B single-engine aerobatic aircraft flying out of Sabadell Airport and operating in visual flight conditions providing up to 8 seconds of hypogravity for each parabola. Aside from biomedical experiments being conducted, different student teams have flown in parabolic flights in the framework of the international contest 'Barcelona Zero-G Challenge', and have published their results in relevant symposiums and scientific journals. The platform can certainly be a good testbed for a proof-of-concept before accessing other microgravity platforms, and has proved to be excellent for motivational student campaigns.
\end{abstract}

\section{Keywords}

Parabolic flights, aerobatics, microgravity, hypogravity, hypergravity.

\section{Introduction}

European scientists have a wide range of possibilities for conducting their experiments in hypogravity conditions; from drop towers (Kufner et al., 2011) to sounding rockets (Dannenberg, 2011), as well as satellites or the International Space Station (ISS). All these facilities provide flight opportunities for their diverse research interests. Aside from these well-known opportunities, parabolic flights have been conducted for a long time as another way of performing short-time duration experiments and technical demonstrations. Aircraft parabolic flights provide up to 23 seconds of reduced gravity and are used for conducting short investigations in Physical and Life Sciences, both for senior researchers and for international student experimentation and motivation.

The European Space Agency (ESA), the “Centre National d'Etudes Spatiales” (CNES, French Space Agency) and the "Deutsches Zentrum für Luft- und Raumfahrt e.V." (DLR, German Aerospace Center) have used the Airbus A300 ZERO-G from Novespace for research experiments in microgravity. This French company, a spin-off from CNES, has been conducting a large amount of parabolic flights for many years (Pletser, 2004). Among these, seven ESA Student campaigns (Callens et al., 2011) and two joint partial-g parabolic flight campaigns (Pletser et al., 2012) have taken place, in the latter case providing partial gravity similar to that present on the surface of the Moon and Mars. In 2014, the long successful Airbus A300 ZERO-G was withdrawn, and in early 2015 a new Airbus A310 ZERO-G entered into operation with which new parabolic flight campaigns are being conducted (Pletser et al., 2015). Parabolic flights are the only current flight opportunity besides Chinese spacecraft and ISS for medical research experimentation on human subjects in microgravity. 
These parabolic flights are useful for conducting preparatory tests. Those tests prepare the experiments to be conducted in future space missions at more sophisticated facilities. They can also be used after a space mission to retest or provide confirmation of previous results. Nevertheless, the short period of hypogravity provided in these flights is often sufficient to obtain meaningful results that can be included in subsequent experiments. Briefly, these aircraft maneuvers allow experimenters to get results by running their own scientific experiments within a relatively low cost framework, and in the case of Europe, from a nearby European airfield, usually in Bordeaux, France.

Since 2008, a series of test flights have been performed in Barcelona, Spain,in order to assess the suitability of smaller single-engine aerobatic aircraft for hypogravity experimentation (Brigos, et al. , 2014a). These parabolic flights are operated by the Aeroclub Barcelona-Sabadell, the second largest Aeroclub in Europe and the oldest and largest Flight School in Spain. Between 2008 and 2011, a non-commercial joint venture was established for scientific collaboration between this Aeroclub and the Universitat Politècnica de Catalunya (UPC). The objective was to first test these small aircraft as a possible facility for conducting parabolic flights. The Aeroclub operates out of Sabadell Airport, a general aviation airfield located $20 \mathrm{~km}$. from Barcelona, where on average more than $70 \%$ of the time the weather allows visual flight operations (VFR). Regular commercial flights do not operate from this airfield. While it is a fully controlled airport, only Flight Schools, private and charter operations are undertaken there, thus leaving free time for practising and implementing test flights. It is the only Aeroclub in Spain that has a fully operational Aerobatic Team. Aerobatic aircraft can be especially interesting for performing parabolic flights, as they are already certified for carrying out such specific maneuvers. Moreover, they have been designed for performing maneuvers which are unusual for a commercial plane. Provided that the operations are conducted within the specified range in the Manual of the aerobatic plane, no modifications are needed to conduct the maneuvers required for a parabolic flight. The Aerobatic Team regularly trains at a specified area within the outskirts of the airfield, so that no specific allowance is required for performing such parabolic flights in the surrounding area.

\section{Objectives and features of small aerobatic aircraft parabolic flights}

As has been proved and is now well established (Pletser et al., 2004), a large aircraft provides researchers with a microgravity environment during a short period of time lasting approximately 20 seconds. This period is preceded and followed by a short period of hypergravity of 1.8 to $2 \mathrm{~g}$ of approximately 20 seconds either side of the hypogravity phase. The sequence can be repeated a number of times within a particular flight, usually 30 , so that the experiment can be repeated in a single day. Parabolic flight objectives pursued by the space agencies, and in particular by the European Space Agency are multifold, including both scientific, technical goals, as well as being motivational for students or outreach to the general public (Callens et al., 2011; Pletser et al., 2015).

As reported by our team, after the initial tests were conducted with a CAP10B aircraft (Brigos et al., 2014a), these small aerobatic aircraft are capable of providing short periods of hypogravity of up to 8 seconds, preceded and followed by a peak of hypergravity up to $3.2 \mathrm{~g}$ 's between 3 and 5 seconds. The quality of the hypogravity provided, measured as the residual acceleration during the hypogravity phase, is approximately an order of magnitude above that obtained with larger aircraft. This is due to limitations such as higher sensitivity to wind gusts, vibrations or the manual operation of the maneuvers. After an initial period of flight tests and optimization of the technique with a simulator and an on-board display, a figure of merit of $0.05 \mathrm{~g}$ of residual acceleration was obtained.

Acceleration levels are typically in the order of 0.05 to $0.1 \mathrm{~g}$ in the Z-axis (aircraft floor to ceiling direction), while accelerations along the aircraft X-axis (aft to front) and transversal Y-axis (right to left) are typically between $0.005 \mathrm{~g}$ and $0.05 \mathrm{~g}$. As an example, Figure 1 shows residual acceleration measured in the cockpit during a typical parabola. 
Figure 1. Residual acceleration measured during a typical parabola with a single-engine aerobatic aircraft.

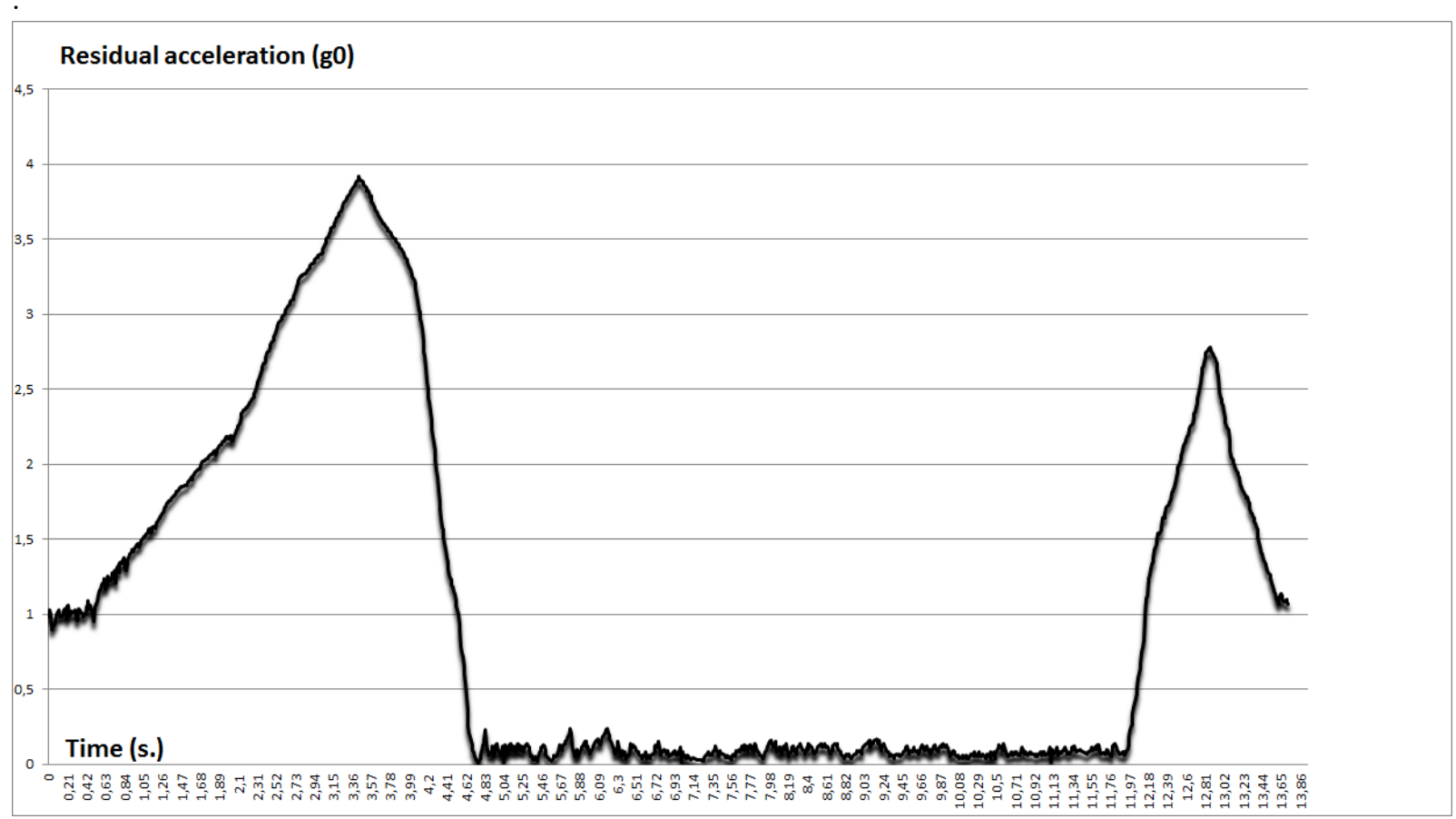

The objectives of parabolic flights with an aerobatic single-engine aircraft are:

1. Scientific

- $\quad$ To study different processes in which abrupt changes of gravity workload are applied. In particular hyper $(3-3.5 \mathrm{~g})$ to hypogravity $(0.05 \mathrm{~g})$, and hypo to hypergravity periods.

- To analyse transient phenomena that may occur after short periods of hyper and hypogravity.

- To allow experiments for testing the equipment in a real parabolic flight, with the opportunity to manually interact with the equipment and provide a proof-of-concept before accessing other microgravity research platforms.

- If the experiment can be run in less than 8 seconds of exposure to hypogravity, and the residual acceleration of $0.05 \mathrm{~g}$ is acceptable, then quantitative and qualitative measurements can be made, thus providing meaningful data. The parabolic flight can provide 10-15 parabolas in a single flight, and weather permitting the procedure can be repeated in a single day.

- In regard to human physiology experiments in which and the hypo and hypergravity environment plays a role, the facility enables different subjects to test the scientific hypotheses, one by one on board.

2. Technological

- Assessment of technological equipment behaviour in a hyper and hypogravity environment with abrupt changes in a tiny environment.

- Safety assessment of experiments and technological demonstrations within a parabolic flight aircraft cockpit. 
- Training of wannabe or future astronauts for foreseen private or public space missions.

\section{Educational and outreach}

- Allowing students to conduct hands-on experiments in a real weightlessness experience.

- Increasing their interest for studying Science, Technology, Engineering and Mathematics (STEM) syllabus, in particular in the aerospace field.

- Providing students from different backgrounds and nationalities with the opportunity of working as a team with a common goal, while interacting with space professionals.

- Raising public interest in space research.

- Creating the opportunity for students to write and present their space research in relevant journals and congresses, and also to further apply to the space agencies educational programs.

Weather permitting, a local VFR flight is conducted in less than 20 minutes from the airfield to the area where the maneuvers are performed. The manoeuvres typically start at an altitude of 1,000m AGL (Altitude above Ground Level), rising up to $1,200 \mathrm{~m}$ AGL. A typical flight will consist of 10 to 15 parabolas and the total duration of the flight until landing will be approximately one hour. Only one experimenter may accompany the pilot on each flight, but the flight may be repeated many times during the day, which enables different researchers to fly on board and conduct their experiments. The maneuvers are manually performed by licensed aerobatic pilots. This particular aircraft is a twin-seater, thus enabling an experimenter to travel on board and conduct the experiments manually, although the aircraft may also carry automated experiments (Figure 2).

Figure 2. CAP10B single-engine aerobatic aircraft used for performing parabolic flights.

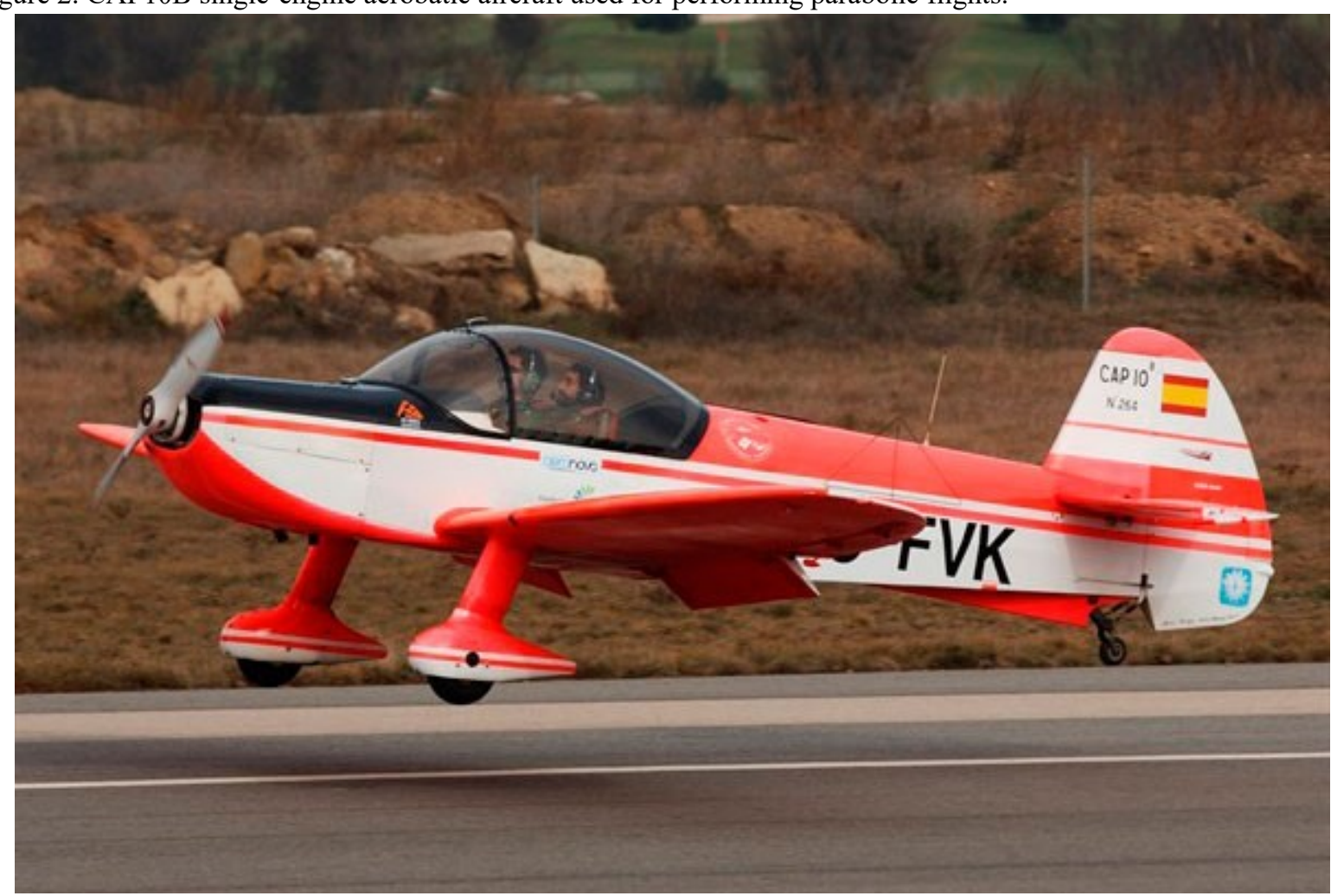

Small payloads of up to $25 \mathrm{~kg}$ with a size of less than $50 \mathrm{~cm} \times 50 \mathrm{~cm} \times 50 \mathrm{~cm}$ can be operated by the experimenter in the aircraft cabin. In addition, the experimental equipment may be also attached to the aircraft 
structure. In this case, only experiments which are not vibration-sensitive (in the range of $0-25 \mathrm{~Hz}$ ) would be suitable. On-board residual accelerations sensed by measurement equipments attached to the aircraft lateral structure are typically above $0.05 \mathrm{~g}$, due mainly to the spinning of the helix. Apart from test flights, four research campaigns and three educational student campaigns have been performed since 2009. A typical research campaign lasts for a full day and consists of up to 6 local flights with an operator and an experiment on board. If weather conditions vary and prevent the aircraft from flying in VFR conditions in the area where the manoeuvres are performed, then the flight should be postponed, which imposes a limitation on these operations. However, the experimenter is able to reach the flight manoeuvre area in less than 30 minutes from the time he is safely seated in the aircraft before takeoff and the experiment is ready for flight. A JAR (European Joint Aviation Regulations) Class II medical certificate or equivalent, and a flight surgeon consultation briefing are required before the experimenter is allowed to fly. A safety briefing is conducted before the flight, and technical consultation is provided for the researchers as well as a limited civil insurance provided by the flight operator, the Aeroclub Barcelona-Sabadell.

\section{3.- Educational and research campaigns conducted in Barcelona}

These parabolic flights are an excellent opportunity for university students to conduct real hands-on experiments on space science. Since the beginning of operations we have been inviting international student teams to propose and actually fly their experiments on board this aircraft. Space agencies like ESA have a long and successful record of providing such opportunities. Other microgravity platforms like the ZARM Drop Tower from Bremen (Germany) or the Swedish rocket platform REXUS/BEXUS (Dannenberg, 2011) also organize international contests for university students on a regular basis. All these activities, among others carried out outside Europe, are important for attracting youthful talent to the exciting world of space research, and retaining it.

In our case, a student campaign consists of between two and six local flights on which one student performs his or her experiment on board during every flight. A team also composed of two to six students can therefore conduct their experiments over the course of a single day. These educational campaigns are known as the "Barcelona Zero-G Challenge", an international contest aimed at motivating students to conduct research in this area. The first such Challenge was carried out in 2010 in a workshop held during the International Space University Summer Space Program (ISU SSP). The workshop consisted in setting up groups of international, interdisciplinary and intercultural teams to design a zero-g experiment which complied with small aircraft requirements. After a peer-review selection, a team of five students, mentored by Prof. Gilles Clément from ISU, flew their experiment in November 2010 in Barcelona. This experiment was entitled 'Reversible Images in Space', the aim of which was to test the deconditioning of the inner ear prioceptive system by measuring the brain's change of perception while in the hypogravity period (Figure 3 ). 
Figure 3. Reversible Images provide interesting clues about the deconditioning of the innear ear prioceptive system in microgravity.

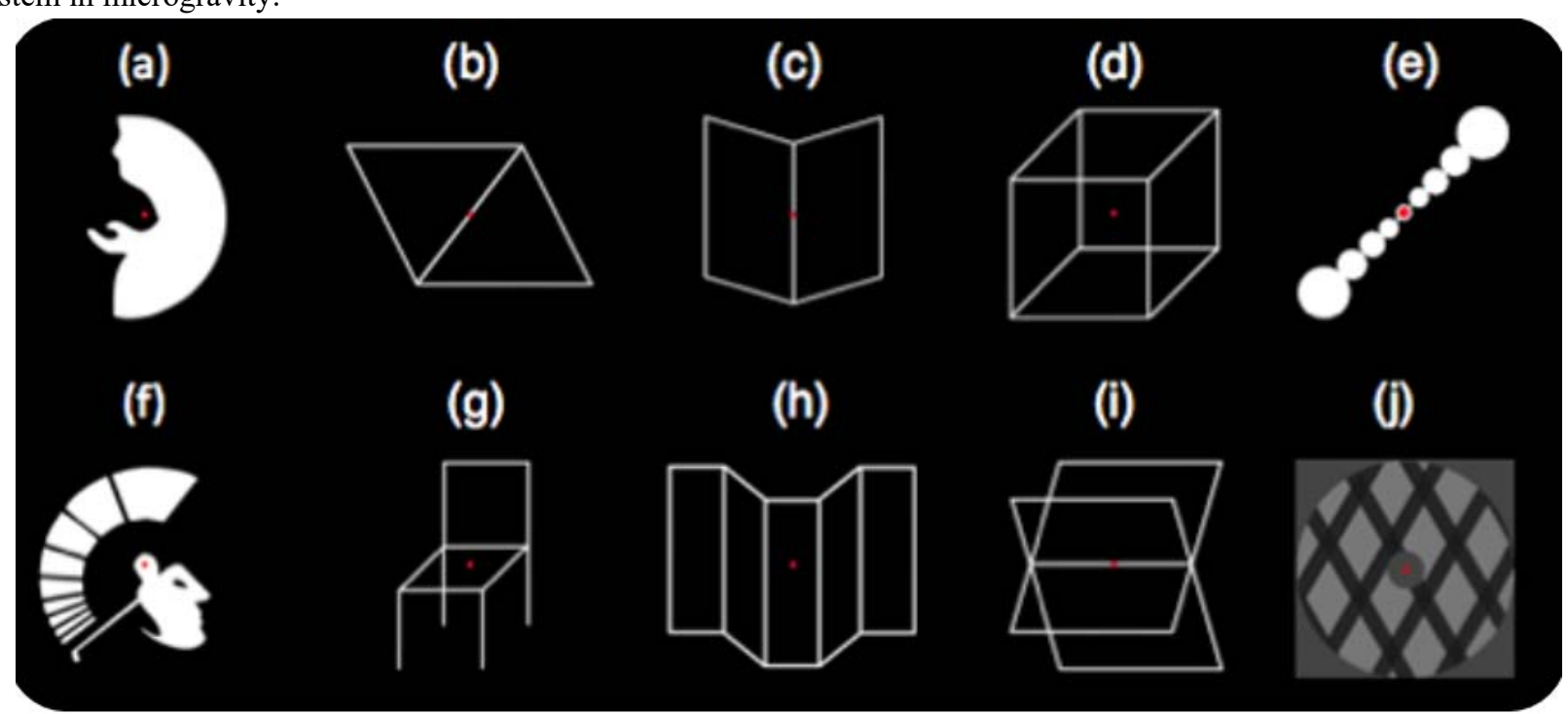

Three students from Canada, one from the United Kingdom and one from France tested the software that was later put into operation in the ISS by astronauts. The results were subsequently published in Plos One (Clément et al., 2015) and some of the same students participated in the analysis and in drawing up the manuscript. This first parabolic flight campaign with aerobatic aircraft was made available to the general public thanks to a local TV broadcast and national newspapers.

A second Barcelona ZeroG Challenge was conducted in 2011. Three students from Uruguay, Spain and Canada composed the winning team, which was mentored by Prof. Nandu Goswami. They performed their experiment "Mental arithmetics during hypogravity" in November 2011. The selection was subject to a strict peer-review process conducted by European Low-Gravity Research Association (ELGRA) senior researchers. The results were later published (Osborne et al., 2014) and provided evidence to show that mental arithmetic while exposed to hypogravity may be considered as a countermeasure for avoiding cardiovascular deconditioning during long space missions. This second student campaign held in Barcelona also received media attention and reached the general public.

In 2014, a third Barcelona ZeroG Challenge was announced, providing an opportunity for two student teams to conduct their experiment on board an aerobatic aircraft. This time the selection was performed under a strict peerreview process with both ELGRA and ESA Education members taking an active part in it. The winning team was composed of two undergraduate students, Valentina Boccia from Italy and Anja Schuster from Germany, who presented their results at the ELGRA Symposium 2015 (Schuster et al., 2015), with Anja Schuster receiving a Students Award from ELGRA for her Poster Presentation (Figure 4). 
Figure 4. Winners of the Barcelona ZeroG Challenge 2014 while preparing their experiment just before flight.

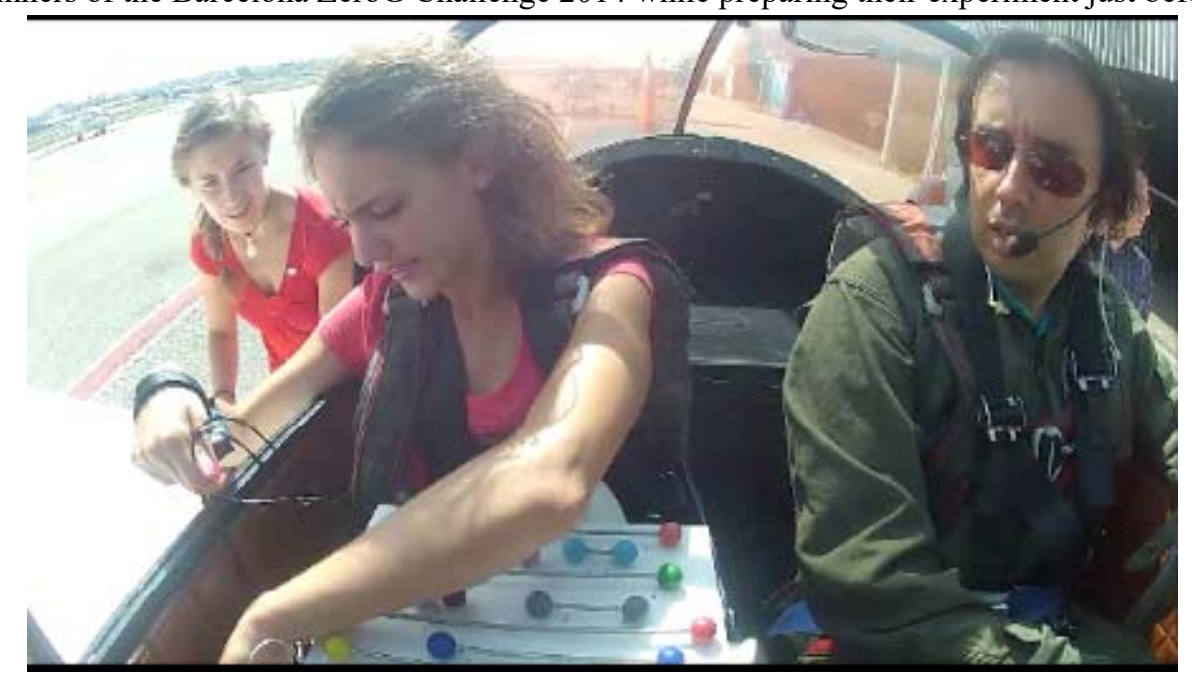

A new edition of this contest is currently in preparation, with the winners expected to fly their experiment in late 2016. Notably, space youth associations such as Euroavia and Space Generation Advisory Council have been active in spreading the word throughout the world about these opportunities.

Educational opportunities are also provided locally to students from our own university. An educational tutorial has been drawn up based on these experiments. This manual contains an introduction to space physiology and explains how the data was obtained and why it was useful, as well as hands-on material consisting of simulation software that students can use to see what changes may happen to the human body when exposed to long-term scenarios, like a long expedition to the Moon or a trip to Mars. The material was tested by engineering students who had almost no previous understanding of medical concepts, and it can equally be used by life sciences students with no knowledge of simulation techniques. A final survey and an evaluation of the students' work were carried out in order to assess the impact of this activity.

The students were required to work out what changes were important and what implications the data had for the hypothesis of the experiment, as well as proposing future research lines. They received a one-hour introductory tutorial to the workshop and two hours of class work, and were given four days to submit their work. All student teams presented their work on time, and the evaluation ranged from fairly good to excellent for all teams. Students qualified with a $3.8+/-0.4$ for the activity (1-boring, 5 -exciting) and provided some comments, such as 'the activity was the most original of my studies' or 'I also wish to take part in the experiments'.

Furthermore, Engineering students belonging to both Master and Graduate courses at our university, the UPC, are offered the possibility of designing such experiments (Azemà, 2014) within the restrictions of our platform, or of analyzing the mechanics and on-flight behavior of our small aircraft.

In addition to student participation in these activities, parabolic research flights have also been performed. Six of these research campaigns have been conducted since 2009, most of them in the field of biomedical studies. For example, researchers from the Universitat Rovira-Virgili recently studied cardio and neurological changes in healthy subjects when exposed to abrupt changes in gravity load (Ruiz et al., 2015). Researchers from our University, the UPC, have also recently analyzed the influence of wind gusts on the gravity quality provided by this particular aircraft (Brigos, et al., 2014b). The platform is permanently open to European researchers within the framework provided by ELGRA for experimentation in parabolic flight, with a flight profile that is notably different from that provided by a larger aircraft.

\section{4.- Present and future of single-engine Aerobatic parabolic flights.}


As operators of these parabolic flights, the Aeroclub Barcelona-Sabadell provide European researchers with the opportunity of participating in these research flights, free of charge, as part of a collaborative research endeavour. The cost of a typical campaign with the CAP10B flight is estimated at 50,000 euros for six flights consisting of one experiment and one subject on board the aircraft over a period of two days, with up to 75 parabolas. This figure includes ownership and maintenance of the aircraft and is supported by the commercial activities of the Aeroclub, which hires the aircfraft to associates and to members of the public for leisure purposes, as well as to members of the Flight School. This relatively low cost of the operations enables us to provide the necessary research and educational opportunities within a restricted budget. The purpose and aim of the Aerobatic parabolic flights described herein is not to compete with other large aircraft from Space agencies, as these aircraft have several limitations. These limitations are: the small cockpit size; the lack of electrical plugs for experiments; sensitivity to wind gusts; less hypogravity time per parabola; limited control of the residual acceleration during this reduced gravity period, and a more aggressive flight profile for the experimenters. However, these parabolic flights have proven capable of yielding meaningful data for scientific research as well as a test-bed for accessing other microgravity platforms. Finally, we would like to emphasize the importance of international scientific collaboration for providing an optimal framework for educational and scientific experimentation.

\section{5.- Conclusions}

This project was first begun seven years ago. Analysis of the outcomes of these activities as reported in this paper show that the platform has proven to be excellent for motivational campaigns as well as providing a test-bed for a proof-of-concept before accessing other microgravity platforms. Throughout this time, many students and senior researchers have had the opportunity of conducting space research in parabolic flights from Barcelona, and they have reported their results at relevant scientific symposiums and in leading journals. We expect to continue these operations in the foreseeable future, thanks to the optimization of the manual technique required for performing such maneuvers and the positive response received from the European scientific community. The highly encouraging feedback from students with hands-on experience from our parabolic flights, as well as their initial advances in the field of the space research, all confirm that our initial objectives have been fulfilled.

\section{Acknowledgements}

Many people have contributed over the years to these successful activities of Barcelona parabolic flights with singleengine aerobatic aircraft. In particular, the authors wish to thank all colleagues from the Aeroclub BarcelonaSabadell and Universitat Politècnica de Catalunya (UPC) who have contributed to this venture. We are especially grateful to the pilots of the Barcelona-Sabadell Aeroclub Aerobatic Team for their skill in performing the maneuvers. We are likewise grateful to all the students and flight participants for their support and contribution. The voluntary involvement of ELGRA and ESA Education members, with Natacha Callens, Francisco J. Medina and Ricard Gonzalez-Cinca as coordinators of the student projects peer-review, also deserve our specific acknowledgement. Thanks are also due to Vladimir Pletser for fruitful discussions.

\section{Bibliography}

Azemà M., "Study of the fused deposition modeling behavior under microgravity conditions", Master Thesis, Universitat Politècnica de Catalunya UPC (2014).

Brigos M., Perez-Poch A., Alpiste F., Torner J., "Parabolic flights with single-engine aerobatic aircraft: flight profile and a computer simulator for its optimization", Microgravity Science and Technology, 26 (4) 229-239, (2014a). 
Brigos M., Perez-Poch A., Torner J., Alpiste F., Lázaro R., "Influence of wind gusts on microgravity quality in parabolic flights with single-engine aerobatic aircraft". Proceedings of the $65^{\text {th }}$ International Astronautical Congress. Paper \# IAC-14-A2.3.9 (2014b).

Callens, N., Ventura-Traveset, J., De Lophem, T.L., Lopez De Echazarreta, C., Pletser, V., Van Loon, J., "ESA parabolic flights, drop tower and centrifuge opportunities for university students". Microgravity Science and Technology 23 (2), 181-189 (2011).

Clément G., Allaway, H., Demel M., Golemis A., Kindrat A., Melinyshyn A., Merali T., Thirsk R., "Long duration spaceflight increases depth ambiguity of reversible perspective figures", Plos One 10(7): e0132317 (2015), doi: 10.1371/journal.pone.0132317

Dannenberg, K., "Swedish space activities- an overview tith a focus on ballons and rockets." Proceedings of the 200th ESA Symposium on European rocket and balloon programmes and related research. ESA Special publications, 700, 33-35 (2011).

Kufner, E., Blum J., · Callens N., · Eigenbrod Ch., Koudelka O., Orr A., Rosa C.C., Vedernikov A., Will S., Reimann J., Wurm G., "ESA's Drop Tower Utilisation Activities 2000 to 2011”. Microgravity Science and Technology, 23 (4), 409-425 (2011).

Osborne J.R., Alonsopérez M.A., Ferrer D., Goswami N., González D.V., Moser M., Grote V., García-Cuadrado G., Perez-Poch A., "Effect of Mental Arithmetic on heart rate responses during Parabolic Flights: the Barcelona Zero-G Challenge”. Microgravity Science and Technology, 26 (1), 11-16 (2014).

Pletser V., "Short duration microgravity experiments in physical and life sciences during parabolic flights: the first 30 ESA campaigns", Acta Astronautica, 55 (10), 829-854 (2004).

Pletser V., Winter J., Bret-Dibat T., Friedrich U., Clervoy J., Gharib T., Gai F., Minster O., Sundblad P., "The First Joint European Partial-G Parabolic Flight Campaign at Moon and Mars Gravity Levels for Science and Exploration" ,Microgravity Science and Technology, 24 (6), 383-395 (2012).

Pletser V., Rouquette S., Friedrich U., Clervoy, Jean-François, Gharib T., Gai F., Mora C. "European parabolic flight campaigns with Airbus ZERO-G: Looking back at the A300 and looking forward to the A310." Advances in Space Research, 56 (2015) 1003-1013.

Ruiz X., Sáez N., Gavalda J., Perez-Poch A., "On the cardio-neurological changes generated on a healthy adult male by short abrupt variations of the gravity load”, Proceedings of the Elgra Symposium and General assembly 2015. Elgra News 31, p. 120 (2015).

Schuster A., Boccia V., Perez-Poch A., González D.V., “ Estimation of relative distance between two objects in microgravity conditions during parabolic flight", Proceedings of the Elgra Symposium and General assembly 2015. Elgra News 31, p.165 (2015). 